\title{
Analysis of the Clinical Effect and Complication Rate of Emergency Surgery for Severe Chest Trauma with Traumatic Shock
}

\author{
Sakarie M Hidig, Jie Yang, Xiaokaiti Yibulayin \\ Emergency Surgery, The First Affiliated Hospital of Xinjiang Medical University, Xinjiang 830011, China
}

\begin{abstract}
Objective: To analyze the clinical effect and complication rate of emergency surgery for severe chest trauma with traumatic shock. Method: A total of 100 patients with severe chest trauma and traumatic shock admitted to our hospital from November 2017 to November 2018 were randomly selected. All the patients were divided into the reference group (50 cases, elective surgical treatment) and the study group (50 cases, emergency surgical treatment) according to the difference in surgical treatment time. At the same time, the effect of surgical treatment, incidence of complications (organ failure, respiratory distress syndrome, empyema, pulmonary infection) and clinical indicators (recovery time of respiratory function, mortality) of patients in the two groups were compared and analyzed. Consequence: The total effective rate of clinical efficacy in the control group and the treatment group was $98 \%$ and $86 \%$, so treatment group was significantly higher than the control group. The incidence of adverse reactions in the control group and the treatment group was $4 \%$ and $12 \%$, so treatment group was significantly lower than the control group, and the recovery time and mortality of respiratory function in the treatment group were lower than those in the control group, with significant differences in all comparisons $(\mathrm{P}<0.05)$. Conclusion: Emergency surgery for patients with severe chest trauma and traumatic shock has a significant therapeutic effect, which can effectively reduce the risk of postoperative complications, improve the prognosis of patients and reduce the short-term mortality of patients.
\end{abstract}

Keywords: Severe Chest Trauma; Traumatic Shock; Emergency Surgery; Elective Surgery

Severe trauma refers to a series of life-threatening injuries at the site of injury, such as open pneumothorax, massive hemorrhage of viscera, severe brain injury, severe fall injury, spinal cord injury, severe pelvic injury and rupture of viscera, which all be called severe trauma and have a fatal risk ${ }^{[1]}$.A series of injuries, such as pelvic injury and internal organ rupture, can be called severe trauma, which may be fatal ${ }^{[1]}$. Severe chest trauma and traumatic shock refers to the syndrome caused by damage to body tissues caused by external forces, resulting in insufficient effective circulating blood volume, microcirculation disorder, accompanied by trauma, etc. Severe chest trauma and traumatic shock are critical and critical diseases, and surgery is the first choice of treatment in clinical practice. However, most patients adopt conservative treatment, including drug stabilization and elective surgery. Although conservative treatment can also play a certain therapeutic effect, it will lead to serious adverse consequences in patients with serious illness due to delayed treatment ${ }^{[2]}$. However, emergency surgery refers to a surgical method that is considered necessary for urgent patients to be treated as soon as possible after evaluation by specialists, which is commonly associated with various acute diseases such as trauma, acute abdomen and massive hemorrhage ${ }^{[3]}$. In this paper, the clinical effect of emergency surgery for severe chest trauma with traumatic shock and its impact on the incidence of complications were studied, specifically as follows:

\section{Objects and methods}

\subsection{Objects}

Our hospital was included in the study from May 2018 to May 2019, with a total of 100 patients with severe chest trauma and traumatic shock. All patients were divided into the reference group (26 males and 24 females, with an average age of $39.58 \pm 8.58$ years) and the treatment group (27 males and 23 females, with an average age of $39.85 \pm 8.17$ years). According to different operative time, there was no statistically significant difference in general data between the two groups $(\mathrm{P}>0.05)$. At the same time, all patients and their families volunteered to participate in this study after knowing the content and standards of this study in detail. All subjects had no allergic reactions to the drugs used in this study, and the ethics Committee of our hospital has fully understood this study and approved it. 


\subsection{Methods}

\subsubsection{Reference group}

Patients in the reference group were selected for elective surgical treatment. After admission, patients were intubated to ensure smooth breathing of patients, and routine treatments such as cleaning and suturing of patients' wounds were given. Patients were transferred to intensive care unit and selected for surgical treatment when their condition was stable.

\subsubsection{Research group}

Patients in the study group were selected for emergency surgical treatment. After admission, the green channel was opened to lead the patients to improve preoperative relevant examinations, endotracheal intubation was administered to ensure smooth breathing of the patients, and the wound surface of the patients was sutured. Meanwhile, CT scanning was performed on the patients simultaneously to determine the degree of injury of the patients, and the surgical plan was formulated according to the examination results. After the operation, the patients were given routine anti-infection and other conventional drugs, paid close attention to the postoperative recovery of the patients, and reduced the risk of lung infection and organ failure.

\subsection{Observation index}

Statistical analysis of the two groups of patients with surgical treatment effect; Recovery: After surgical treatment, the clinical symptoms of the patients completely disappeared, without abnormal vital signs. Significant effect: After surgical treatment, clinical symptoms were improved and postoperative recovery was good. Effective: After surgical treatment, the clinical symptoms were partially improved and the postoperative recovery was slow. No effect: no improvement or aggravation of clinical symptoms after operation; Total effective rate $=($ cure + obvious effect + effective rate)/all cases $\times 100 \%$; Incidence of complications $($ organ failure, respiratory distress syndrome, empyema, pulmonary infection) and patient clinical indicators (recovery time of respiratory function, mortality).

\subsection{Statistical analysis}

The data in this study were statistically analyzed using software SPSS22.0. Measurement data were represented by (), t-test was performed, and count data were represented by $\mathrm{N}(\%)$. Comparison with chi-square test, $\mathrm{P}<0.05$ was considered statistically significant.

\section{Consequence}

\subsection{Treatment effect analysis of two groups of patients}

The total effective rate of clinical efficacy was $98 \%$ in the control group and $86 \%$ in the treatment group, which was significantly higher than that in the control group. The difference was statistically significant $(\mathrm{P}<0.05)$, as shown in Table 1 :

Table 1. Comparison of therapeutic effect between treatment group and control group [N, (\%)]

\begin{tabular}{ccccccc}
\hline Group & $\begin{array}{c}\text { Cases } \\
\text { number }\end{array}$ & Cure & Apparent effect & Valid & Invalid & Effective rate \\
\hline Control group & 50 & $5(10.00)$ & $11(22.00)$ & $27(54.00)$ & $7(14.00)$ & $43(86.00)$ \\
Treatment group & 50 & $9(18.00)$ & $27(54.00)$ & $13(26.00)$ & $1(2.00)$ & $49(98.00)$ \\
$\chi^{2}$ & - & 2.658 & 21.732 & 16.333 & 9.783 & 9.783 \\
P & - & 0.103 & 0.000 & 0.005 & 0.002 & 0.002 \\
\hline
\end{tabular}

\subsection{Analyzed incidence of adverse reactions in the two groups}

The incidence of adverse reactions in the control group and the treatment group was $4 \%$ and $12 \%$, so treatment group was significantly lower than the control group. The difference was statistically significant $(\mathrm{P}<0.05)$, as shown in Table 2 :

Table 2. Comparison of adverse reactions between treatment group and control group [N, (\%)]

\begin{tabular}{ccccccc}
\hline Group & $\begin{array}{c}\text { Cases } \\
\text { number }\end{array}$ & Organ failure & $\begin{array}{c}\text { Respiratory distress } \\
\text { syndrome }\end{array}$ & pyothorax & $\begin{array}{c}\text { pulmonary } \\
\text { infection }\end{array}$ & $\begin{array}{c}\text { Effective } \\
\text { rate }\end{array}$ \\
\hline $\begin{array}{c}\text { Control } \\
\text { group }\end{array}$ & 50 & $3(6.00)$ & $2(4.00)$ & $1(2.00)$ & $1(2.00)$ & $6(12.00)$ \\
$\begin{array}{c}\text { Treatment } \\
\text { group }\end{array}$ & 50 & $1(2.00)$ & $1(2.00)$ & $0(0.00)$ & $0(0.00)$ & $2(4.00)$ \\
$\chi^{2}$ & - & 2.083 & 0.687 & 2.020 & 2.020 & 4.348 \\
$P$ & - & 0.149 & 0.407 & 0.155 & 0.155 & 0.037 \\
\hline
\end{tabular}

\subsection{Analysis clinical index of two groups patients}

The recovery time of respiratory function and mortality of patients in the treatment group were lower than those in the control group, and the difference was statistically significant $(\mathrm{P}<0.05)$, as shown in Table 3:

Table 3. Comparison of clinical indicators between treatment group and control group $(\mathrm{x} \pm \mathrm{s}) /[\mathrm{n},(\%)]$

\begin{tabular}{cccc}
\hline Group & Cases number & Recovery time of respiratory function $(\mathrm{d})$ & Death rate $(\%)$ \\
\hline Control group & 50 & $3.17 \pm 0.58$ & $5(10.00)$ \\
Treatment group & 50 & $1.59 \pm 0.37$ & $1(2.00)$
\end{tabular}




\begin{tabular}{cccc}
$\mathrm{t} / \chi^{2}$ & - & 16.240 & 5.674 \\
$\mathrm{P}$ & - & 0.000 & 0.017 \\
\hline
\end{tabular}

\section{Discussion}

With the rapid development of China's social economy, the replacement of transportation means, so that the incidence of safety accidents increased year by year. As an accidental disease caused by external impact, severe chest trauma can seriously damage the chest of patients. Due to the sudden and critical condition, which the mortality rate of patients with severe chest trauma is extremely high, making it one of the current clinical critical diseases ${ }^{[4]}$.

At present, the treatment of this sudden disease is mostly conservative elective surgery or emergency surgery ${ }^{[5]}$ On admission, respiratory tract cleaning and endotracheal intubation are routinely performed to ensure the patient's normal breathing. Traditional conservative elective surgery usually USES conventional drugs to stabilize patients' condition, relieve patients' pain and improve their shock symptoms. However, it is very easy to delay the best time of treatment for patients, which can not fundamentally eliminate the threat of the disease to the lives of patients. Once the disease recurs, the death rate will increase several times. In addition, with the improvement of living standards and the improvement of people's health awareness, conservative elective surgery can no longer meet the treatment needs of patients with severe chest trauma ${ }^{[6]}$.

For emergency surgery, preoperative examination should be carried out in time after admission to clarify organ damage, assess the patient's operation tolerance, formulate reasonable operation plan according to the patient's specific situation, and carry out the operation as soon as possible, so as to carry out the operation treatment in the shortest time. In addition, patients should pay close attention to the changes of their vital signs after surgery, correct their failing organs and internal circulation, and give prevention and control intervention for postoperative complications to reduce the risk of postoperative complications. Emergency surgery can effectively improve the effect of surgical treatment and reduce the short-term mortality of patients by timely judging patients' conditions, clarifying the risk factors of patients' life safety, providing patients with rescue time and saving patients' injured organs. The results also showed that patients with emergency operation treatment efficiency is as high as $98 \%, 86 \%$, significantly higher than elective surgery patients emergency surgical treatment for patients with postoperative adverse reaction rate was only $4 \%$, significantly lower than the $12 \%$ patients with elective surgical procedures, and emergency surgery in the treatment of patients with respiratory function recovery time and mortality due to elective surgery patients.

To sum up, emergency surgery for patients with severe chest trauma and traumatic shock has a significant therapeutic effect, which can effectively reduce the risk of postoperative complications, improve the prognosis of patients and reduce the short-term mortality of patients.

\section{References}

1 Xing Youxuan, Zhang Junyong. Analysis of the clinical effects and the incidence of complications in emergency surgical treatment of severe chest trauma and traumatic shock [J]. China Health and Nutrition, 2019, 029(005): 121-122.

2 Li Jinghan. Clinical effect observation and safety analysis of emergency surgery for severe chest trauma complicated with traumatic shock [J]. Modern Diagnosis and Treatment, 2018, 029(022): 3670-3671.

$3 \mathrm{Wu}$ Junhua, Wen Jianwen. Discussion on the clinical method and significance of emergency surgery for patients with severe chest trauma and traumatic shock [J]. Heilongjiang Medical Science, 2018, 42(11): 1095.

4 Liu Keyuan. Comparison of the application effect of emergency surgery and elective surgery in patients with chest trauma combined with shock [J]. Modern Practical Medicine, 2019, 031(006): 774-775.

5 Yan Kaixuan, Wu Yanmei. Clinical value analysis of emergency surgery for severe chest trauma with traumatic shock [J]. Electronic Journal of Clinical Medicine Literature, 2020, 430(09): 74-75.

6 Zhao Xinang, Li Arafune, Huo Yanwei, et al. Clinical value of emergency surgery for severe chest trauma with traumatic shock [J]. Guizhou Medicine, 2019, 043(008): 1303-1305.

7 Wang Yubiao, Lin Hai, Zhang Lijuan, et al. Analysis and Discussion of emergency surgery in 25 cases of severe chest trauma with traumatic shock [J]. Diabetes World Education (Early Part), 2019, 016(004): 179-180. 